\title{
Depression following graft-versus-host disease in a patient with acute lymphoblastic leukaemia: A case report
}

\author{
KONSTANTINOS TSAMAKIS ${ }^{1}$, CHRISTOPH MUELLER ${ }^{2,3}$, PANAGIOTIS TSIRIGOTIS ${ }^{4}$, \\ DIMITRIOS TSIPTSIOS $^{5}$, CHARALAMPOS TSAMAKIS $^{6}$, EMMANOUIL CHARAKOPOULOS ${ }^{1}$, \\ CHISTOPHIS CHARALAMPOUS ${ }^{1}$, DEMETRIOS A. SPANDIDOS ${ }^{7}$, ATHANASIOS DOUZENIS ${ }^{1}$, \\ CHARALABOS PAPAGEORGIOU $^{8}$, IOANNIS LIAPPAS ${ }^{8}$ and EMMANOUIL RIZOS $^{1}$
}

\begin{abstract}
${ }^{1}$ Second Department of Psychiatry, School of Medicine, University General Hospital 'Attikon', 12462 Athens, Greece; ${ }^{2}$ King's College London, Institute of Psychiatry, Psychology and Neuroscience, London SE5 8AF;

${ }^{3}$ South London and Maudsley NHS Foundation Trust, London, UK; ${ }^{4}$ Haematology Clinic, School of Medicine, University General Hospital 'Attikon', 12462 Athens, Greece; ${ }^{5}$ South Tyneside and Sunderland NHS Foundation Trust, Sunderland SR47TP, UK; ' Department of Dermatology, School of Medicine, University General Hospital 'Attikon', 12462 Athens; ${ }^{7}$ Laboratory of Clinical Virology, Medical School, University of Crete, 71003 Heraklion;

${ }^{8}$ First Department of Psychiatry, Athens University Medical School, Eginition Hospital, 11528 Athens, Greece
\end{abstract}

Received November 1, 2019; Accepted December 17, 2019

DOI: $10.3892 / \mathrm{mco} .2019 .1970$

\begin{abstract}
There is increasing evidence to suggest an association between depression and inflammation, with patients suffering from immune mediated-disorders exhibiting higher levels of depression. Inflammation in depression is a potential target for the development of novel treatment strategies. The present study presents a clinical case in which a patient with an underlying inflammatory condition acutely developed a severe depressive episode resulting in a sudden, dramatic change in their clinical picture. This case, with no similar case reports being in the literature thus far, at least to the best of our knowledge, highlights the increasing consideration that there may be a causative role between neuro-inflammation and depression. This study reports the case of a 40-year-old male with acute lymphoblastic leukaemia (ALL) and no previous psychiatric history, who developed an acute onset of a severe depressive episode in the context of the immune-mediated graft-versus-host disease (GVHD). GVHD is a complication of allogeneic hematopoietic cell transplantation, which the patient had undergone, for the treatment of his ALL. The rapid onset of depression could be explained by the neuroinflammatory processes occurring in GVHD. This provides a clinical example for the possible role of the immune system in depression, and clinicians should be aware of this association.
\end{abstract}

Correspondence to: Associate Professor Emmanouil Rizos, Second Department of Psychiatry, School of Medicine, University General Hospital 'Attikon', Rimini 1, Chaidari, 12462 Athens, Greece

E-mail: erizos@med.uoa.gr

Key words: depression, inflammation, graft-versus-host disease, acute onset, leukaemia

\section{Introduction}

There has been increased focus in recent literature on the possible role of inflammation in the pathogenesis of depression $(1,2)$. Depression has been described as an inflammatory state, as depressed patients have been found to exhibit high blood levels of C-reactive protein (CRP) and inflammatory cytokines [interleukin (IL)-1, IL-6 and tumour necrosis factor (TNF- $\alpha)](3,4)$, which can be normalized following the initiation of treatment with anti-depressants (5). Individuals with chronic immune-mediated inflammatory diseases, such as rheumatoid arthritis, exhibit a higher prevalence of depression (6), whilst anti-depressants may alter immune function and can potentially suppress immune-mediated disorders, such as acute graft-versus-host disease (GVHD) (7). Neuroinflammation is a potential target for novel treatments in depression, with the option for more personalized treatment in subgroups of patients, possibly identified through biological immune markers predicting an improved treatment response (2), or in the context of treatment-resistant depression (8).

Acute lymphoblastic leukaemia (ALL) is a cancer of the lymphoid line of blood cells in which the uncontrolled proliferation of lymphoblasts (of B- or T-cell origin) occurs in the bone marrow, peripheral blood and/or other organs (9). It is characterized by the development of large numbers of immature lymphocytes and causes a loss of energy, pale skin colour, fever, easy bleeding or bruising, enlarged lymph nodes and bone pain. Allogeneic hematopoietic cell transplantation (HCT) is a widely used therapy for a variety of malignant haematological diseases, including ALL (10).

GVHD is a common complication of allogeneic HCT (11). Acute GVHD occurs within the first 100 days of transplantation and presents with manifestations of the skin, gastrointestinal mucosa and liver. Chronic GVHD develops after day 100 and 
consists of an autoimmune syndrome directed toward multiple organs. GVHD occurs when immune cells transplanted from a non-identical donor recognize the recipient's cells as foreign due to major histocompatibility complex (MHC) molecule mismatch. As a result, an immune reaction is initiated; this subsequently causes disease in the host (12). The pathogenesis of GVHD is a complex, multistep process. It is primarily a T-cell mediated disease. In order to activate the graft T-cells, two cellular signalling events must occur. First of all, there must be an interaction between the T-cell receptor (TCR) of the donor T-cells and the MHC of the host's cells (13), and for the second signal, a cell-to-cell contact is necessary (14). Once the T-cells are activated, a variety of pro-inflammatory cytokines are released (15). According to previous studies, when a MHC mismatched hematopoietic cell transplantation is performed in laboratory mice, CD4 and CD8 cells produce IL-1, IL-3 and IL-7 (16), as well as granulocyte-macrophage colonystimulating factor (GM-CSF), TNF- $\alpha$ and $\gamma$-interferon (17). These cytokines eventually activate other T-cells or other cell types, such as monocytes and natural killer (NK) cells. In the end, activated transplanted T-cells damage the host's cells.

This study hereby presents the case report of a 40 -yearold male with the sudden onset of depressive symptoms after developing GVHD post-HCT.

\section{Case report}

A 40-year old male with no previously recorded personal or family psychiatric history was admitted to the Haematology Clinic of 'Attikon' Hospital (Athens, Greece) with recurrent ALL and subsequently underwent allogeneic bone marrow transplantation. At 3 months after transplantation, he developed an extensive maculopapular skin rash, which gradually progressed to widespread hyperpigmentation of the skin. As a result, he was re-admitted in the Haematology Clinic of 'Attikon' Hospital, where a clinical diagnosis of GVHD was established, which was confirmed histologically with a skin biopsy. He was successfully treated with systemic corticosteroids, without any observed change in the mental and emotional state of the patient.

Over the following months, he received treatment with systemic steroids on a gradually reducing regime. He was monitored as an outpatient at the Haematology Department on a regular basis and remained fairly stable clinically. At 15 months after the transplantation, he developed an unexplained fever, for which he was admitted to the Haematology Clinic. After receiving treatment with intravenous antibiotics, the patient became apyrexial. Of note, during this whole period, and up to the day before his expected discharge, there were no changes in his mental state and his mood remained euthymic.

One day prior to his planned discharge, he began experiencing a non-specific, vague abdominal disturbance. In addition, there was an unexpected, acute onset of a depressive effect and low mood. Increasingly, over the next few days, he stopped eating and his night sleep became severely disturbed. He stopped communicating (with no evidence of frank aphasia) and was reluctant to see his wife and children. His motivation and energy were markedly reduced, and he began expressing a fear of death. Of note, until this marked change in his mental state, he was described as a very jovial, hopeful and sociable person and there was no report of any depressive symptoms in the recent past.

Despite extensive investigations (including abdominal CT scans) and liaison with other medical specialties, no specific cause for the abdominal disturbance was found. Although his ALL remained in remission, his mood continued to deteriorate; as a consequence, consultation by the Liaison Psychiatric team was requested.

Upon psychiatric assessment, he was described as profoundly low in mood with pronounced anhedonia (i.e., not taking pleasure in things he used to). He had developed biological symptoms of severe depression: His energy was significantly reduced; his sleep was severely disturbed, and he presented with a lack of reduced appetite and marked weight loss. In addition, he expressed hopelessness and helplessness, and reported a new onset of passive death wishes and suicidal thoughts, although without a suicidal plan. He was however, well-orientated and scored 30/30 in the Mini Mental State Examination (MMSE); therefore, the possibility of hypoactive delirium and organic brain cause were excluded.

At 2 weeks after the onset of the ascribed symptoms, he was given a clinical, working diagnosis of a severe depressive episode without psychotic symptoms. His symptom severity was assessed with the Hamilton Depression (HAM-D) Rating Scale; his score was 25 , which is indicative of severe depression. Treatment with the serotonin-norepinephrine reuptake inhibitor (SNRI), duloxetine, was initiated and instructions for the further monitoring of his mental state and risk were given. Duloxetine was titrated up to $60 \mathrm{mg}$ daily with partial response; over the next 3 weeks, the death wishes subsided, although he remained very low in mood and the biological symptoms of depression continued. His symptom severity was assessed again with the HAM-D rating scale, and his score was 17 at the time.

The patient continued to complain of vague abdominal disturbance, for which, no definite organic cause could be established. More than a month after the onset of the symptoms, he developed localized abdominal pain in the lower left quadrant; a repeat abdominal CT scan revealed intestinal leakage, for which he was taken to the operating theatre for explorative laparotomy. Although the leakage was successfully dealt with, he unfortunately developed sepsis and passed away 2 days later in the Intensive Care Unit. The cause of the intestinal leakage was inconclusive. Of note, at the time of death and during the days preceding his death, no marked change had been observed in his mental state; he remained severely depressed.

\section{Discussion}

The case presents the rapid onset of severe depressive symptomatology in the context of GVHD in a patient with no previous psychiatric history. Inflammation is increasingly considered as an etiological factor for depression $(2,18,19)$. It is worth noting that the patient whose case is presented herein had developed skin hyperpigmentation, which is a post- inflammatory manifestation of GVHD (20). Given that the patient was in a good mental state not only premorbidly, but also during the several months post-HCT (which makes the possibility of 
his depression being reactive to the psychological burden of his diagnosis and treatment less likely), it is possible that the GVHD-induced neuro-inflammation triggered the acute onset of a severe depressive episode.

In observational studies, chronic GVHD has been recognized as a risk factor for depression following HCT (21). Mechanistically, cytokines released during GVHD could cause depression. It is well-established that, in chronic medical conditions which are accompanied by inflammation, such as cancer, infections and autoimmune diseases, there is an increased risk of depression; patients who suffer from these conditions tend to develop depression more frequently than the general population (22). Additionally, pro-inflammatory agents can induce depressive symptoms (2), whilst elevated levels of inflammatory markers have been recognized as future predictors of depression (23). This is further supported by the fact that anti-inflammatory treatment has anti-depressant effects and anti-inflammatory drugs are likely to particularly benefit depressed patients who exhibit evidence of inflammation (19).

Pro-inflammatory cytokines can activate central nervous system circuitry and evoke adverse neurobehavioral and affective responses, including depressed mood, fatigue, sleep disturbance, decreased activity, anorexia and neurocognitive dysfunction (24). HCT recipients are at particularly high risk for developing inflammation-related impairments due to the 'cytokine storm' associated with GVHD. A previous study on allogeneic transplant recipients tracked both inflammatory cytokines and several symptoms during the initial 30 days post-transplantation; it was found that increases in IL-6 and sTNF-R1 levels were associated with a deterioration of fatigue, poor appetite, pain, drowsiness, dry mouth and disturbed sleep (24). Pro-inflammatory cytokines may affect the brain directly, through the penetration of the blood-brain barrier, and indirectly, through the regulation of the electrical activity of the vagus nerve, the stimulation of hormone production from peripheral tissues (25) and the migration of peripheral monocytes to the central nervous system (CNS) (26). Depression may be the result of the cytokine-induced reduction in the expression of the brain-derived neurotrophic factor (BDNF), a neurotrophin involved in neuroplasticity (27). The reduced concentration of BDNF may be attributed to the ability of cytokines to increase the extracellular concentrations of glutamate in the CNS. The subsequent binding of glutamate to $\mathrm{N}$-methyl-D-aspartate (NMDA) receptors leads to nerve cell death and the decreased expression of BDNF.

Furthermore, cytokines can lead to reduced concentrations of the monoamines, serotonin (5-HT), dopamine (DA) and norepinephrine (NE), in the synaptic cleft. More specifically, cytokines increase the activity of amine reuptake pumps (28), decrease the expression of enzymes involved in the synthesis of monoamines through increase of oxidative stress (29) and increase the breakdown of the serotonin precursor, tryptophan (30). Moreover, it is worth mentioning that IL-1, IL-6 and TNF- $\alpha$ have been consistently proven to stimulate the hypothalamic-pituitary-adrenal (HPA) axis, the anatomical structures of which are abundant in cytokine receptors (31). This is of particular importance as the overstimulation of the HPA axis and a deficient negative glucocorticoid feedback mechanism play a central role in the pathogenesis of melancholic, major depression (32).
Recent studies have indicated a potentially causal role for cytokines in depression (19), which is in concordance with our hypothesis about the inflammation-induced rapid onset depression in the case presented herein. The understanding of this association is of utmost importance as it can lead to the development of novel treatment strategies for depression; cytokine modulators have been suggested as potentially novel drugs for depression in subjects with chronic inflammation (19).

In conclusion, the case described herein presented a rapid onset of severe depressive symptomatology in the context of GVHD and highlights that the underlying neuro-inflammatory processes taking place in GVHD could trigger a depressive episode. Clinicians should thus be vigilant for the possible development of mood disorders in the context of GVHD, even in patients without previous psychiatric history.

Understanding the mechanisms through which inflammation may affect brain function to induce and/or perpetuate mood disorders could inform future clinical practice and may lead to the development of novel, better targeted and more personalized treatment options.

\section{Acknowledgements}

Not applicable.

\section{Funding}

No funding was received.

\section{Availability of data and materials}

Data sharing is not applicable to this article as no new data were created or analysed in this study.

\section{Authors' contributions}

ER and PT were members of the clinical teams who treated the patient and were major contributors to the writing of the manuscript. KT, CM, CT, CC and EC were major contributors to the writing of the manuscript. DT, AD, DAS, CP and IL substantially revised the manuscript. All the authors (KT, CM, PT, DT, CT, EC, CC, DAS, AD, CP, IL and ER) took part in discussions pertaining to the presentation of this case report, and assisted in the finding of relevant reference citations pertaining to this topic. All authors have read and approved the final manuscript.

\section{Ethics approval and consent to participate}

The Ethics Approval and approval for the use of patient data were obtained by the Scientific Committee of 'Attikon' Hospital (Athens, Greece) with the approval no. EBA 111/2602-2018.

\section{Patient consent for publication}

The patient, before passing away, had fully consented to the sharing of the data of his clinical condition and treatment, that could be used for all scientific purposes, which includes relevant publications of his case in scientific journals. 


\section{Competing interests}

DAS is the Editor-in-Chief for the journal, but had no personal involvement in the reviewing process, or any influence in terms of adjudicating on the final decision, for this article. All the other authors declare that they have no competing interests.

\section{References}

1. Osimo EF, Baxter LJ, Lewis G, Jones PB and Khandaker GM Prevalence of low-grade inflammation in depression: A systematic review and meta-analysis of CRP levels. Psycho Med 49: 1958-1970, 2019.

2. Köhler O, Krogh J, Mors O and Benros ME: Inflammation in depression and the potential for anti-inflammatory treatment. Curr Neuropharmacol 14: 732-742, 2016.

3. Dinan TG: Inflammatory markers in depression. Curr Opin Psychiatry 22: 32-36, 2009.

4. Howren MB, Lamkin DM and Suls J: Associations of depression with C-reactive protein, IL-1, and IL-6: A meta-analysis. Psychosom Med 71: 171-186, 2009.

5. Janssen DG, Caniato RN, Verster JC and Baune BT: A psychoneuroimmunological review on cytokines involved in antidepressant treatment response. Hum Psychopharmacol 25: 201-215, 2010.

6. Dickens C, McGowan L, Clark-Carter D and Creed F: Depression in rheumatoid arthritis: A systematic review of the literature with meta-analysis. Psychosom Med 64: 52-60, 2002

7. Gobin V, Van Steendam K, Fevery S, Tilleman K, Billiau AD, Denys D and Deforce DL: Fluoxetine reduces murine graft-versus-host disease by induction of T cell immunosuppression. J Neuroimmune Pharmacol 8: 934-943, 2013.

8. Kiraly DD, Horn SR, Van Dam NT, Costi S, Schwartz J, Kim-Schulze S, Patel M, Hodes GE, Russo SJ, Merad M, et al Altered peripheral immune profiles in treatment-resistant depression: Response to ketamine and prediction of treatment outcome. Transl Psychiatry 7: e1065, 2017.

9. Al Ustwani O, Gupta N, Bakhribah H, Griffiths E, Wang E and Wetzler M: Clinical updates in adult acute lymphoblastic leukemia. Crit Rev Oncol Hematol 99: 189-199, 2016.

10. Matsukuma KE, Wei D, Sun K, Ramsamooj R and Chen M: Diagnosis and differential diagnosis of hepatic graft versus host disease (GVHD). J Gastrointest Oncol 7: S21-S31, 2016.

11. Jacobs JM, Fishman S, Sommer R, Sereno I, Fenech A, Jankowski AL, et al: Coping and modifiable psychosocial factors are associated with mood and quality of life in patients with chronic graft-versus-host disease. Biol Blood Marrow Transplant 25: 2234-2242, 2019.

12. Ferrara JL, Levine JE, Reddy P and Holler E: Graft-versus-host disease. Lancet 373: 1550-1561, 2009.

13. Sette A, Alexander J and Grey HM: Interaction of antigenic peptides with MHC and TCR molecules. Clin Immunol Immunopathol 76: 168-171, 1995.

14. Yang Y and Wilson JM: CD40 Ligand-dependent T Cell activation: Requirement of B7-CD28 signaling through CD40 Science 273: 1862-1864, 1996.

15. Jadus MR and Wepsic HT: The role of cytokines in graft-versushost reactions and disease. Bone Marrow Transplant 10: 1-14, 1992.

16. Dean RM, Fry T, Mackall C, Steinberg SM, Hakim F, Fowler D, Odom J, Foley J, Gress R and Bishop MR: Association of serum interleukin-7 levels with the development of acute graft-versushost disease. J Clin Oncol 26: 5735-5741, 2008.

17. Korngold R and Sprent J: T cell subsets and graft-versus-host disease. Transplantation 44: 335-339, 1987.
18. Enache D, Pariante CM and Mondelli V: Markers of central inflammation in major depressive disorder: A systematic review and meta-analysis of studies examining cerebrospinal fluid, positron emission tomography and post-mortem brain tissue. Brain Behav Immun 81: 24-40, 2019.

19. Kappelmann N, Lewis G, Dantzer R, Jones PB and Khandaker GM: Antidepressant activity of anti-cytokine treatment: A systematic review and meta-analysis of clinical trials of chronic inflammatory conditions. Mol Psychiatry 23: 335-343, 2018.

20. Strong Rodrigues K, Oliveira-Ribeiro C, de Abreu Fiuza Gomes S and Knobler R: Cutaneous graft-versus-host disease: Diagnosis and treatment. Am J Clin Dermatol 19: 33-50, 2018.

21. Syrjala KL, Langer SL, Abrams JR, Storer B, Sanders JE, Flowers ME and Martin PJ: Recovery and long-term function after hematopoietic cell transplantation for leukemia or lymphoma. JAMA 291: 2335-2343, 2004.

22. Felger JC and Lotrich FE: Inflammatory cytokines in depression: Neurobiological mechanisms and therapeutic implications. Neuroscience 246: 199-229, 2013.

23. Gimeno D, Kivimäki M, Brunner EJ, Elovainio M, De Vogli R, Steptoe A, Kumari M, Lowe GD, Rumley A, Marmot MG, et al: Associations of C-reactive protein and interleukin- 6 with cognitive symptoms of depression: 12-year follow-up of the Whitehall II study. Psychol Med 39: 413-423, 2009.

24. Costanzo ES, Juckett MB and Coe CL: Biobehavioral influences on recovery following hematopoietic stem cell transplantation. Brain Behav Immun 30: 68-74, 2013.

25. Guyon A, Massa F, Rovère C and Nahon J-L: How cytokines can influence the brain: A role for chemokines? J Neuroimmunol 198: 46-55, 2008.

26. D'Mello C, Le T and Swain MG: Cerebral microglia recruit monocytes into the brain in response to tumor necrosis factoralpha signaling during peripheral organ inflammation. J Neurosci 29: 2089-2102, 2009.

27. Calabrese F, Rossetti AC, Racagni G, Gass P, Riva MA and Molteni R: Brain-derived neurotrophic factor: A bridge between inflammation and neuroplasticity. Front Cell Neurosci 8: 430, 2014.

28. Zhu C-B, Lindler KM, Owens AW, Daws LC, Blakely RD and Hewlett WA: Interleukin-1 receptor activation by systemic lipopolysaccharide induces behavioral despair linked to MAPK regulation of CNS serotonin transporters. Neuropsychopharmacology 35: 2510-2520, 2010

29. Neurauter G, Schröcksnadel K, Scholl-Bürgi S, SpernerUnterweger B, Schubert C, Ledochowski M and Fuchs D: Chronic immune stimulation correlates with reduced phenylalanine turnover. Curr Drug Metab 9: 622-627, 2008.

30. Maes M, Leonard BE, Myint AM, Kubera M and Verkerk R: The new '5-HT' hypothesis of depression: Cell-mediated immune activation induces indoleamine 2,3-dioxygenase, which leads to lower plasma tryptophan and an increased synthesis of detrimental tryptophan catabolites (TRYCATs), both of which contribute to the onset of depression. Prog Neuropsychopharmacol Biol Psychiatry 35: 702-721, 2011.

31. Turnbull AV and Rivier C: Regulation of the HPA axis by cytokines. Brain Behav Immun 9: 253-275, 1995.

32. Dinan TG and Scott LV: Anatomy of melancholia: Focus on hypothalamic-pituitary-adrenal axis overactivity and the role of vasopressin. J Anat 207: 259-264, 2005.

This work is licensed under a Creative Commons Attribution-NonCommercial-NoDerivatives 4.0 International (CC BY-NC-ND 4.0) License. 